Cahiers d'études italiennes

\title{
Seduzione amorosa e seduzione artistica in Retablo di Vicenzo Consolo
}

Tatiana Bisanti

\section{OpenEdition}

\section{Journals}

\section{Edizione digitale}

URL: http://journals.openedition.org/cei/813

DOI: $10.4000 /$ cei.813

ISSN: 2260-779X

\section{Editore}

UGA Éditions/Université Grenoble Alpes

\section{Edizione cartacea}

Data di pubblicazione: 15 septembre 2006

Paginazione: 57-68

ISBN: 978-2-84310-086-4

ISSN: 1770-9571

\section{Notizia bibliografica digitale}

Tatiana Bisanti, «Seduzione amorosa e seduzione artistica in Retablo di Vicenzo Consolo», Cahiers d'études italiennes [Online], 5 | 2006, online dal 15 mars 2008, consultato il 28 mars 2021. URL: http:// journals.openedition.org/cei/813 ; DOl: https://doi.org/10.4000/cei.813 


\title{
SEDUZIONE AMOROSA E SEDUZIONE ARTISTICA IN RETABLO DI VINCENZO CONSOLO
}

\author{
Tatiana Bisanti \\ Universität des Saarlandes - Saarbrücken
}

È solo a partire dal XVI secolo che il verbo sedurre ha acquisito l'accezione oggi comunemente più diffusa, quella di "convincere al rapporto sessuale", entrata nell'italiano per influenza del francese séduire. Il verbo deriva dal prefisso se- "via, a parte" e ducere "condurre", e significa dunque in origine "condurre via, condurre in disparte", ovvero lontano da un percorso diritto e già segnato, dalla retta via (cfr. Battaglia 1961). E proprio il sedurre in tutti i sensi, anche in quello etimologico, è senza dubbio una delle chiavi di lettura più efficaci del romanzo Retablo (1987) di Vincenzo Consolo, un'opera sulla seduzione, ma anche e soprattutto un'opera di seduzione. La seduzione è, in altre parole, l'asse su cui ruota tutta la narrazione, sia dal punto di vista della materia narrata che da quello delle strutture narrative, ossia essa è al tempo stesso oggetto di rappresentazione e operazione in corso. Se da un lato, infatti, il sedurre è tematizzato nel racconto, dall'altro esso costituisce anche il principio strutturale dell'opera: la metafora della seduzione raffigura emblematicamente il processo in cui l'autore coinvolge il lettore, conducendolo su più strade, su diversi percorsi narrativi che si dipartono l'uno dall'altro e si intrecciano a vicenda.

\section{La scrittura della seduzione}

Come suggerisce il titolo, Retablo ha la struttura di un'ancona a vari scomparti: il romanzo si divide - come un trittico - in tre parti (Oratorio, Peregrinazione Veritas), tre percorsi narrativi che raccontano la stessa storia da tre prospettive differenti. Il filone narrativo principale, il denominatore comune che unisce le tre parti del racconto, è una storia di seduzione, la storia dell'amore del frate Isidoro per Rosalia, che viene narrata da tre punti di vista. Nella prima parte, Oratorio, la storia è raccontata dalla pro- 
spettiva di Isidoro, narratore in prima persona. In Peregrinazione, la parte centrale, l'io narrante è quello di Fabrizio Clerici, pittore milanese che Isidoro accompagna nel corso del suo viaggio in Sicilia. La storia di Isidoro si intreccia allora con quella di Clerici, anch'egli amante infelice, in fuga da un amore non corrisposto per la milanese Teresa Blasco, storicamente la nonna di Manzoni, che andrà poi in sposa a Cesare Beccaria. Il capitolo finale, Veritas, è invece una lettera indirizzata ad Isidoro in cui Rosalia racconta la storia dal proprio punto di vista. E come in un retablo sotto gli elementi del polittico c'è una predella, così anche nel romanzo di Consolo al filone narrativo principale si accompagna un altro percorso narrativo, ovvero la storia di un'altra Rosalia, sedotta dal corrotto fra' Giacinto.

Retablo è innanzitutto, come dice Traina (2001: 27), "un romanzo sull'amore deluso", un tema che affiora sempre assai pudicamente negli altri libri di Consolo, mentre qui si fa motore e ragione di tutta l'azione, di tutto il viaggio. Tre sono dunque le storie d'amore principali (ma ad esse si potrebbero aggiungere altri intrecci secondari) su cui si dipana il filo del racconto. La seconda sezione del libro, quella centrale e di gran lunga la più estesa, è il diario di viaggio che Clerici scrive per Teresa Blasco, la donna amata da cui cerca di allontanarsi compiendo la sua "peregrinazione" attraverso la Sicilia. È solo attraverso il "collaudato contravveleno della distanza”, infatti, che Clerici riesce a ritrovare quell'“aura irreale o trasognata" che gli consente di dedicarsi alla scrittura e alla pittura (Consolo 1987: 87). ${ }^{1}$ Quello di Clerici è, per così dire, un caso di seduzione mancata: in fuga da un amore infelice, alla fine del suo viaggio apprenderà che l'amata sta per unirsi in matrimonio con Cesare Beccaria. Nel descrivere un momento di particolare abbandono nel corso del viaggio, la sosta presso i Bagni Segestani, Clerici chiama a raccolta i più celebri miti di seduzione della tradizione letteraria: in particolare quello di Ulisse indotto ad accettare i doni della maga Circe o deciso a resistere al canto seduttore delle sirene, oppure la boccacciana novella di Biancofiore (p. 61-62). La scena del bagno è il momento di seduzione più alto descritto da Clerici, ma è significativo che si tratti di una scena giocata sul puro piano dell'immaginazione: come Ulisse con le sirene, Clerici si fa sedurre solo in parte dal canto delle fanciulle che gli giunge attraverso la parete che separa il bagno degli uomini da quello delle donne. Non è un caso che il pittore non arrivi ad oltrepassare questa parete che divide i due mondi: in

1. Per le successive citazioni da quest'opera si indicherà d'ora in poi solo il numero di pagina. 
quanto artista egli può vivere il momento della seduzione solo a livello immaginario, e sempre mediato attraverso il filtro della letteratura. In questo momento di abbandono sensuale quelli che affiorano alla mente di Clerici sono episodi attinti esclusivamente all'immaginario letterario, quasi a frapporre una distanza rassicurante fra sé e il mondo, ad attutire attraverso lo schermo della finzione la forza dirompente della realtà in tutta la sua concretezza.

Un altro episodio del romanzo è emblematico di come la seduzione dell'arte assolva talvolta ad una funzione di compensazione nei confronti di una seduzione mancata nella vita: in quanto castrato, il maestro di canto don Gennaro non potrà mai sedurre l'adorata Rosalia, e sublima la propria mutilazione attraverso l'arte:

C'est la vie: chi canta non vive e chi vive non canta! [...] siamo castrati tutti quanti vogliamo rappresentare questo mondo: il musico, il poeta, il cantore, il pintore... Stiamo ai margini, ai bordi della strada, guardiamo, esprimiamo, e talvolta, con invidia, con nostalgia struggente, allunghiamo la mano per toccare la vita che ci scorre per davanti. (p. 158)

Ma la classica contraddizione decadentista fra arte e vita viene subito contraddetta, relativizzata e deformata da una lente ironica: con un brusco abbassamento di registro Rosalia interpreta questo allungare la mano alla lettera, e lo riconduce ad un piano ben più concreto e corporeo:

Ha ragione, ha ragione don Gennaro! Così fanno, allungano le mani, questi artisti. Così il vecchio abate Meli, che alla Flora, sbucando avanti a me all'improvviso da un cespuglio, tocca, sospira e va, declamando poesie.

Così il cavalier Serpotta, lo scoltore plastico, quando il marchese mi portò da lui per farmi fare le pose per le statue della Veritas: al posto dello stucco, plasmava me, con insistente mano. (p. 158)

Un'analoga concretezza si ritrova nella storia della seduzione di una giovane di nome Rosalia da parte di fra' Giacinto. Questa narrazione è inserita, come si è detto, a mo' di predella a metà del romanzo e si sovrappone al racconto di Clerici grazie ad un felice espediente meta-narrativo: Clerici scrive infatti il proprio diario di viaggio sul retro di fogli già scritti, che recano sul davanti il racconto della storia di Rosalia (una delle tante Rosalie di Retablo, la cui identità o eventuale parentela con la Rosalia di Isidoro resta peraltro imprecisata). Approfittando dell'ingenuità della giovane, Giacinto si serve della religione come travestimento per le proprie voglie e i propri desideri più licenziosi. La descrizione di questo episodio è pervasa di forte sensualità e arricchita di particolari concreti.

Ma la vicenda amorosa centrale su cui si basa tutto il romanzo è la storia di seduzione di cui sono protagonisti Isidoro e la giovane Rosalia: 
sedotto prima dal cibo offertogli da Rosalia e dalla madre, poi dalle bellezze della giovane, Isidoro si innamora follemente, tanto da essere indotto a derubare il convento per portare soldi alle due donne. Scoperto l'imbroglio, Isidoro è costretto a fuggire e ad allontanarsi dall'amata. Sarà preso a servizio da Clerici, che riconosce in lui la vittima di "uno di quegli amori furenti che non trovano giammai appiglio o risonanza nel cuore del bersaglio al quale disperatamente son puntati. Ma forse questo è mai sempre il destino di qualsivoglia amore" (p. 34). L'amore, secondo Clerici, "è inseguimento vano, è inganno e abbaglio, fuga notturna in circolo e infinita, anelito mai sempre inappagato" (p. 34). La seduzione di Isidoro, nella descrizione che lui stesso ne fa, all'inizio non si discosta molto dai topo $i$ tradizionalmente associati al processo dell'innamoramento. Il fuoco d'amore si accende attraverso gli occhi e gli sguardi, che, conformemente alla tradizione, sono detti "lampi" (p. 18): anche la scelta lessicale rimanda dunque alla lirica medievale. Ed è proprio sui topoi di questa tradizione (sguardi schivi, donna angelicata) che è costruita tutta la scena iniziale della seduzione:

E gli occhi tenea bassi per vergogna, ma da sotto il velario delle ciglia fuggivan lampi d'un fuoco di smeraldo. Mai m'ero immaginato, mai avevo visto in vita mia, in carne o pittato, un angelo, un serafino come lei. (p. 18)

Ma è una tradizione che si rivela subito inadeguata a rendere conto della concretezza dei fatti, e che pertanto viene subito straniata attraverso l'introduzione di particolari ben più materiali e corporei:

Ché Rosalia, lei, a poco a poco aprì la mantellina, mostrò il corpetto, ciocche arricciate del color del rame, le boccole agli orecchi trasparenti; e sorrideva, con le fossette, mostrando a malapena i denti di cagnola. (pp. 18-19)

Il narratore non si dilunga sui particolari; ed è reticente sul seguito, descritto in modo riassuntivo e lapidario: "Breve, la sera appresso conobbi il paradiso. E nel contempo cominciò l'inferno, l'inferno pel rimorso del peccato e per la ruberia del guadagno a danno del convento" (p. 19). ${ }^{2}$ In parte analoghi, ma più concreti, carnali e sensuali, i toni con cui Rosalia descrive gli stessi eventi dal proprio punto di vista: "mai prima di quella notte io provai il paradiso, né mai per l'innante proverò. [...] E dopo quella notte, non volli più lavarmi, sciogliere l'essenza, disperdere l'odore tuo sparso nel mio corpo" (p. 156). Isidoro dal canto suo paragona la

2. Questo passo sembra far eco alla reticenza e allusività di Francesca nel dantesco: "Quel giorno più non vi leggemmo avante" (Inferno, V, 138). 
figura dell'amata a quella della statua di Santa Rosalia, protettrice di Palermo. La sua passione per la giovane si confonde con il culto e l'adorazione della Santa:

uguale a la Santuzza, sei marmore finissimo, lucore alabastrino, ambra e perla scaramazza, màndola e vaniglia, pasta martorana fatta carne. Mi buttai ginocchioni avanti all'urna, piansi a singulti, a scossoni della cascia, e pellegrini intorno, «meschino, meschino...», a confortare. Ignoravano il mio piangere blasfemo, il mio sacrilego impulso a sfondare la lastra di cristallo per toccarti, sentire quel piede nudo dentro il sandalo che sbuca dall'orlo della tunica dorata, quella mano che s'adagia molle e sfiora il culmo pieno, le rose carnacine di quel seno... E il collo tondo e il mento e le labbruzze schiuse e gli occhi rivoltati in verso il Cielo... (p. 16)

E proprio il mito di Santa Rosalia, con la sua caratteristica fusione di misticismo e carnalità, sottolinea magistralmente questa sintomatica unione di amore divino e profano: nella descrizione del rapimento di Isidoro venerazione della santa, godimento estetico, desiderio erotico ed esperienza estatica diventano tutt'uno. L'annichilamento di Isidoro è totale nel momento in cui riconosce le fattezze dell'amata Rosalia nell'immagine allegorica della Veritas scolpita dal cavalier Serpotta:

Davanti a una di quelle dame astanti, una fanciulla bellissima, una dìa, m’arrestai. VERITAS portava scritto sotto il piedistallo. Era scalza e ignude avea le gambe, su fino alle cosce piene, dove una tunichetta trasparente saliva e s'aggruppava maliziosa al centro del suo ventre, e su velava un seno e l'altro denudava, al pari delle spalle, delle braccia... Il viso era vago, beato, sorridente... Mi sentii strozzare il gargarozzo, confondere la testa, mancare i sentimenti. Aprii disperato le ganasce e lanciai un urlo bestiale. - Rosaliaaa!... - urlai, e caddi a terra tramortito. (p. 24)

Questa la descrizione dello stesso fatto dal punto di vista di Clerici: "davanti a la Verità, divenne matto: crollato in terra, si contorse, schiumò, lacerossi gli abiti, la faccia, quindi nel vico si diede a piangere, a urlare come un forsennato" (p. 149). Lo stato di sopraffazione che colpisce Isidoro di fronte all'immagine di Rosalia rimanda ai sintomi della sindrome di Stendhal. C’è, infatti, un legame inscindibile fra esperienza estetica ed estasi amorosa. Ė attraverso il procedimento dell'ekphrasis, familiare alla tradizione letteraria medievale, che si realizza nella scrittura di Consolo quel legame fra seduzione amorosa e seduzione artistica. Come hanno rilevato molti critici, in Consolo scrittura e arti figurative sono strettamente collegate fra loro. ${ }^{3}$ La seduzione della scrittura consoliana

3. "In Consolo il linguaggio letterario cerca di rivaleggiare con la pittura" (Geerts 2000: 308); "In Retablo c'è l'esplicitazione dell'esigenza della citazione iconografica: il «retablo» appartiene alla pittura ma è anche "teatro», come nell'intermezzo di Cervantes" (Traina 2001: 130). 
agisce soprattutto sull'aspetto visivo, gioca con l'immagine, lo stimolo ottico. È un fenomeno che si ritrova già nel Sorriso dell'ignoto marinaio, dove la storia si sviluppa proprio a partire da un quadro, il Ritratto di ignoto di Antonello da Messina. L'affastellarsi delle sollecitazioni visive è una delle componenti fondamentali di quello che è stato definito il neobarocco di Consolo. Non occorre insistere sul legame fra scrittura e pittura in Retablo. Esso è annunciato già programmaticamente nel titolo, e sottolineato inoltre dalla scelta dei personaggi: quello fittizio di Fabrizio Clerici è, non a caso, pittore, e rimanda direttamente all'omonimo personaggio reale (1913-1993), il pittore amico di Consolo e autore dei cinque disegni che accompagnano la versione a stampa del romanzo. Del resto non è solo sulla stimolazione visiva che gioca il barocco consoliano: il discorso letterario di Retablo fa leva su un vero e proprio coinvolgimento dei sensi (oltre alla vista, come si è già detto, anche l'udito, l'olfatto e soprattutto il gusto). ${ }^{4} \mathrm{Si}$ tratta, pertanto, di una scrittura che può essere senz'altro definita "seduttiva", in quanto mette in gioco le tecniche e i meccanismi tipici della seduzione.

\section{La seduzione della scrittura}

La seduzione in Retablo non è dunque solo rappresentazione e tematizzazione del desiderio e delle sue modalità di manifestazione, ma soprattutto tecnica narrativa in atto, procedimento attraverso cui il testo conquista il suo destinatario. Il romanzo, infatti, irretisce il lettore nelle trame molteplici della narrazione, lo sorprende e lo avvince con la sfaccettatura e la pluralizzazione delle voci e delle prospettive, lo affascina con la sua lingua musicale, con un enunciato che, prima di appellarsi al significato, gioca innanzitutto sull'esplorazione delle potenzialità sonore, sfruttate al massimo attraverso il ritmo incalzante delle enumerazioni e un uso altissimo e intenso dell'allitterazione.

Si è già visto come la stessa storia di amore e seduzione, quella di Isidoro e Rosalia, sia raccontata da tre persone e pertanto a partire da tre prospettive diverse: ognuno narra la propria verità, il romanzo è costruito su una serie di percorsi indecidibili, tutti altrettanto validi. L'intrecciarsi e il sovrapporsi dei vari racconti conferisce al romanzo una struttura paradigmatica, associativa, verticale. È interessante notare come la pluralizza-

4. Si veda ad esempio il contributo già citato di Geerts (2000) sui sapori della cucina consoliana e i numerosi passi del romanzo dedicati ai piaceri culinari (fra l’altro, p. 45, 51, 56-57). 
zione dei punti di vista e delle verità possibili avvenga proprio a partire dalle varie figure di donne sedotte o seduttrici, che sembrano confluire in un volto solo: quando infatti Clerici si accinge a disegnare un profilo di donna che nelle sue intenzioni dovrebbe ritrarre l'amata Teresa Blasco, don Vito vi riconosce la Rosalia per amor della quale aveva ucciso il seduttore fra' Giacinto, mentre Isidoro è convinto che si tratti della propria Rosalia (p. 87). Il fulcro su cui convergono tutti questi percorsi sono dunque le fattezze, ma soprattutto il nome di Rosalia, personaggio dall'identità fluttuante e incerta. Questo nome viene a definire personaggi di volta in volta diversi e non identificabili l'uno con l'altro, diventando quindi una sorta di nome comune, nome di genere atto a definire una categoria di validità pressoché universale. Rosalia è il nome dell'amata di Isidoro, è la Rosalia del manoscritto, è la donna amata dal cavalier Serpotta e modello della statua raffigurante la Veritas; ma Rosalia è anche la santa venerata a Palermo e oggetto di culto da parte di innumerevoli devoti. Su Rosalia convergono tutti i percorsi di desiderio dei vari personaggi e dello stesso lettore. Tutto ciò è evidente fin dall'incipit, un sapiente esercizio di bravura stilistica tutto costruito sulla scomposizione del nome in Rosa e Lia e sulle suggestioni ed associazioni che ne scaturiscono. ${ }^{5}$ Si penserà in proposito alle innumerevoli valenze attribuite da Petrarca al nome dell'amata Laura, mentre, sul versante moderno, il modello indiscusso è senza dubbio il Nabokov di Lolita, non a caso uno scrittore di seduzione per eccellenza. ${ }^{6}$ Nell'incipit di Retablo il gioco fonico e associativo che prende il via dal nome dell'amata è un brillante esempio delle capacità di seduzione della lingua consoliana:

Rosalia. Rosa e lia. Rosa che ha inebriato, rosa che ha confuso, rosa che ha sventato, rosa che ha roso, il mio cervello s'è mangiato. Rosa che non è rosa, rosa che è datura, gelsomino, bàlico e viola; rosa che è pomelia, magnolia, zàgara e cardenia. Poi il tramonto, al vespero, quando nel cielo appare la sfera d'opalina, e l'aere sfervora, cala misericordia di frescura e la brezza del mare valica il cancello del giardino, scorre fra colonnette e palme del chiostro in clausura, coglie, coinvolge, spande odorosi fiati, olezzi distillati, balsami grommosi. Rosa che punto m'ha, ahi!, con la sua spina vele-

5. È lo stesso Consolo a sottolinearlo: "ho scelto il nome Rosalia per S. Rosalia, ma anche per la scomposizione rosa e lia" (De Martino 1992: 48).

6. Si veda in particolare l'incipit del romanzo, a cui Consolo si è sicuramente ispirato (cfr. in proposito Scuderi 1997: 105):

Lolita, light of my life, fire of my loins. My sin, my soul. Lo-lee-ta: the tip of the tongue taking a trip of three steps down the palate to tap, at three, on the teeth. Lo. Lee. Ta.

She was Lo, plain Lo, in the morning, standing four feet ten in one sock. She was Lola in slacks. She was Dolly at school. She was Dolores on the dotted line. But in my arms she was always Lolita. (Nabokov 1996: 7) 
nosa in su nel cuore.

Lia che m'ha liato la vita come il cedro o la lumia il dente, liana di tormento, catena di bagno sempiterno, libame oppioso, licore affatturato, letale pozione, lilio dell'inferno che credei divino, lima che sordamente mi corrose l'ossa, limaccia che m'invischiò nelle sue spire, lingua che m'attassò come angue che guizza dal pietrame, lioparda imperiosa, lippo dell'alma mia, liquame nero, pece dov'affogai, ahi!, per mia dannazione. Corona di delizia e di tormento, serpe che addenta la sua coda, serto senza inizio e senza fine, rosario d'estasi, replica viziosa, bujo precipizio, pozzo di sonnolenza, cieco vagolare, vacua notte senza lume, Rosalia, sangue mio, mia nimica, dove sei? (pp. 15-16)

Tutto il passo è giocato sull'enumerazione e sull'accumulazione delle associazioni evocate dal nome Rosalia, associazioni che rimandano soprattutto a percezioni sensoriali. È proprio da questo insistito richiamo ai sensi che risulta evidente come il lettore si trovi qui coinvolto in un raffinatissimo gioco di seduzione. Si è già parlato dell'estrema forza visiva e dell'icasticità della scrittura consoliana. Ad essa si aggiungono qui sensazioni uditive create soprattutto dalle sonorità allitteranti del testo e da un andamento ritmico più vicino alla poesia che alla prosa. Si veda ad esempio la prima frase, in cui vengono elencati gli effetti della rosa sull'io narrante: i cinque elementi che la compongono possono essere letti come versi (i primi quattro saranno senari) a rima alternata ( $a b a b a$, se si considera confuso e roso come rima siciliana). Non manca il richiamo alle percezioni tattili (la spina della rosa che punge, e più avanti l'impulso a toccare la statua della santa), ma soprattutto a quelle olfattive e gustative. Sarà soprattutto la prima parte del nome, la rosa, ad evocare odori inebrianti, segno di un processo di seduzione in corso, di un desiderio risvegliato e ancora insoddisfatto. Le immagini suscitate dalla seconda parte del nome, Lia, rimandano invece piuttosto alle sensazioni del palato, ad un piacere che, nel momento in cui viene gustato, si rivela subito veleno, seduzione letale che culmina in un "bujo precipizio, pozzo di sonnolenza, cieco vagolare, vacua notte senza lume". Si noti come le associazioni che prendono il sopravvento alla fine del passo insistano proprio sulla mancanza di luce e sulla cecità, a sottolineare il fatto che alla perdita dell'amata corrisponde la fine delle percezioni dei sensi.

Alle associazioni sensoriali risvegliate dal nome Rosalia si aggiungono quelle culturali e letterarie: da un lato l'effigie della santa venerata a Palermo, rappresentata nell'iconografia popolare con il capo cinto da una corona di rose bianche, dall'altro tutte le suggestioni letterarie legate all'immagine della rosa. La seduzione della scrittura nei confronti del lettore si avvale allora di tutta una serie di trame intertestuali che attraversano il testo e si sviluppano proprio a partire dal motivo della rosa: è lo 
stesso Consolo a mettere in risalto le affinità tra il discorso di Isidoro e Rosalia e il contrasto di Cielo d'Alcamo Rosa fresca aulentissima. ${ }^{7}$ Il primo e l'ultimo quadro sono costruiti sul modello del contrasto. L'esempio di seduzione offerto dalla tradizione sarà in questo caso ironico. Ma non è da escludere nemmeno una suggestione derivante dal Roman de la Rose, storia allegorica di seduzione e conquista amorosa in cui l'amata è rappresentata, appunto, da una rosa (ma la metafora risale del resto già alla tradizione latina). Un altro intertesto di importanza centrale sono I promessi sposi manzoniani: non a caso l'amata di Clerici è Teresa Blasco, nonna di Manzoni; e come per quest'ultimo il Seicento era l'occasione per parlare dell'Ottocento, così per Consolo il Settecento è il filtro attraverso cui raccontare l'epoca attuale, come dimostra del resto l'anacronismo nella scelta del personaggio di Fabrizio Clerici, in realtà, come si è già detto, pittore contemporaneo.

La stratificazione dei piani temporali viene ad aggiungersi alla sovrapposizione dei piani narrativi di cui si parlava prima. Quest'ultima trova la sua realizzazione più evidente nell'espediente dei fogli già scritti da Rosalia sul cui retro Clerici annota i suoi appunti di viaggio. Dice in proposito il narratore:

Sembra un destino, quest'incidenza, o incrocio di due scritti, sembra che qualsivoglia nuovo scritto, che non abbia una sua tremenda forza di verità, d'inaudito, sia la controfaccia o l'eco d'altri scritti. Come l'amore l'eco d'altri amori da altri accesi e ormai inceneriti. (p. 89)

In tal modo Clerici istituisce un parallelo diretto fra scrittura e vicende amorose. E prosegue paragonando l'intersezione delle scritture a un retablo:

E il mio diario dunque ha proceduto [...] come la tavola in alto d'un retablo che poggia su una predella o base già dipinta, sopra la memoria vera, vale a dire, e originale, scritta da una fanciulla di nome Rosalia. Che temo sia la Rosalia amata da don Vito Sammataro, per la quale uccise, e si convertì in brigante. O pure, che ne sappiamo?, la Rosalia di Isidoro. O solamente la Rosalia d'ognuno che si danna e soffre, e perde per amore. (p. 89)

La metafora del retablo equivale qui a quella postmoderna del palinsesto.

7. Cfr. De Martino (1992: 48). Si vedano anche i diretti rimandi lessicali: "Ahi, non ho abènto" (p. 16) richiama il "per te non ajo abento notte e dia" di Cielo d'Alcamo (Segre/ Ossola 1997: 93). L'interesse per Cielo d'Alcamo è documentato inoltre nel romanzo stesso dall'episodio metaletterario dell'incontro di Clerici con l'"Accademia de' Ciulli Ardenti" di Alcamo, dove si discute sull'opportunità di "far crescere la cima sicola da ràdica toscana o puramente far sbocciare in aura toscana la semente sicola” (p. 46). 
L'espediente dei fogli già scritti e riscritti sul retro, che la veste tipografica del romanzo riproduce fedelmente, raffigura in modo concreto e visibile quell'intertestualità di cui si parlava prima e che costituisce uno dei tratti salienti di Retablo. Il lettore può scegliere liberamente quale delle due storie leggere. L'interpretazione è aperta e l'intrecciarsi dei piani narrativi ha come effetto, da un lato, la pluralizzazione dei punti di vista, dall'altro però anche l'universalizzazione del destino individuale. La verità, sebbene inafferrabile, è al tempo stesso universalmente valida: tutti sono accomunati da uno stesso destino, vittime di uno stesso gioco di seduzione. La moltiplicazione dei piani narrativi è, in ultima analisi, solo apparente, perché tutti i percorsi convergono alla fine verso un destino universale che coinvolge tutti, lettore incluso.

L'immagine del retablo compare più volte nel romanzo, secondo la tecnica postmoderna della mise en abyme. La spiccata autoreferenzialità e autoriflessività letteraria di certi passi è finalizzata ad un processo autocritico che si rivolge contro l'arte e le sue capacità di seduzione, contro la sua natura finzionale e fasulla che trae in inganno lo spettatore ingenuo. Tuttavia si tratta di un inganno che si rivela anche benefico, in quanto seduce, ovvero allontana l'ingannato dalla triste realtà, offrendogli consolazione e oblio:

L'invenzione di far veder nel quadro ciò che si vole, dietro ricatto d'essere, se non si vede, fortemente manchevole o gravato d'una colpa, non mi sembrò originar da loro. E mi sovvenni allora ch'era la trama comica de l'entremés del celebre Cervantes, intitolato appunto El retablo de las maravillas, giunto di Spagna in questa terra sicola e dai due fanfani trasferito dalla finzione del teatro nella realitate della vita per guadagnar vantaggi e rinomanza. Io mi chiedei allor, al di là dell'imbroglio di Crisèmalo $\mathrm{e}$ Chinigò, nel vedere quei rozzi villici rapiti veramente e trasportati in altri mondi e vani, su alte sfere e acute fantasie, sopra piani di luce e trasparenze, col solo appiglio d'un quadro informe e incomprensibile e la parola più mielosa e scaltra, io mi chiedei se non sia mai sempre tutto questo l'essenza d'ogni arte (oltre ad essere un'infinita derivanza, una copia continua, un'imitazione o impunito furto), un'apparenza, una rappresentazione o inganno, come quello degli òmini che guardano le ombre sulla parete della caverna scura, secondo l'insegnamento di Platone, e credono sian quelle la vita vera, il reale intero, come l'inganno per la follia dolce de l'ingegnoso hidalgo de la Mancha don Chisciotte, che combatté contra i molini a vento presi per giganti, o per furore tragico d'Aiace che fe' carneficina delle greggi credendola d'Atridi, o come l'illusione che crea ad ogni uom comune e savio l'ambiguo velo dell'antica Maya, velo benefico, al postutto e pietoso, che vela la pura realtà insopportabile, e insieme per allusione la rivela; l'essenza dico, e il suo fine il trascinare l'uomo dal brutto e triste, e doloroso e insostenibile vallone della vita, in illusori mondi, in consolazioni e oblii. Ch'ora accattavano i villani a poco prezzo, ponendo nel cappello piumato di Chinigò il loro obolo. (pp. 55-56)

Il retablo è dunque una metafora dell'arte, della sua inadeguatezza a rap- 
presentare il reale in maniera univoca e attendibile e al tempo stesso del suo grande fascino e potere di seduzione. In un'intervista l'autore ribadisce che "l'idea del retablo è mutuata da Cervantes, il padre del romanzo moderno. In effetti l'arte non rappresenta mai la verità; ma semmai rappresenta la verità ideologica di chi scrive" (De Martino 1992: 40). Quella di Cervantes è, dice Consolo, "un'autocritica dell'arte come illusione... un'ironia su se stessi. L'arte come menzogna. Il Don Chisciotte è il più grande romanzo ironico, un'ironia sui poemi cavallereschi” (De Martino 1992: 48). E altrettanto ironico è il titolo dell'ultimo capitolo di Retablo, Veritas, che è anche il nome della statua allegorica modellata sulle fattezze di Rosalia. La verità che Rosalia rivela alla fine nella lettera in cui confessa il proprio amore per Isidoro non è che una delle possibili varianti della stessa storia di seduzione. Sarà da leggere in chiave ironica la ripetizione della formula "Bella, la verità", che ricorre con cadenza quasi ossessiva nel discorso di Rosalia. Emblematico è anche il passo, citato sopra, dove la statua raffigurante la Veritas viene descritta dalla prospettiva di Isidoro (p. 24): la verità non è altro che una figura di donna seducente e ingannatrice. La seduzione sensuale operata dalla statua rimanda a quella intellettuale esercitata dal concetto di verità: esattamente come la seduzione dei sensi, la seduzione intellettuale della verità si rivela illusoria e fasulla, e viene subito dopo relativizzata attraverso il racconto della stessa storia a partire da altri punti di vista.

Retablo è, in ultima analisi, un romanzo sulla seduzione avvenuta e bruscamente finita (quella di Isidoro e Rosalia), sulla seduzione mancata (quella di Teresa Blasco da parte di Clerici), sulla seduzione dai risvolti tragici (quella di Rosalia da parte di fra' Giacinto, brutalmente ucciso dall'innamorato Don Vito), o sulla seduzione spostata, deviata (la seduzione dell'arte che compensa quella irrealizzabile della carne fra don Gennaro e Rosalia). Ma soprattutto - e in questo consiste la particolarità del romanzo - la seduzione rappresentata va di pari passo con la seduzione in atto esercitata dalla scrittura, che trascina il lettore coinvolgendolo su molteplici piani di lettura. La conseguente relativizzazione della verità artistica fa parte di un processo autocritico che si rivolge non solo contro la "valanga di libri e di libresse privi d'anima, costrutto, lepóre e ragione ch'oggidì invadon biblioteche, botteghe di librai, si spargono pel mondo" (p. 30), ma contro l'arte tutta in generale, e perfino, in particolare, contro lo stesso Retablo consoliano. Così come il retablo ovvero il quadro teatrale seduce e inganna i "rozzi villici rapiti" (p. 55) che lo contemplano, altrettanto fa il romanzo Retablo in quanto prodotto artistico: la mise en abyme è allora al tempo stesso da un lato uno specchio autoriflessivo e autocritico 
che riflette la finzione artistica, dall'altro uno specchio che rifrange la realtà e la riproduce in modo sfaccettato e molteplice, rivelandone in tal modo l'irrimediabile inafferrabilità.

\section{Bibliografia}

Battaglia S., Grande dizionario della lingua italiana, Torino, UTET, 1961 -

Consolo V., Retablo, Palermo, Sellerio, 1987.

De Martino M., Intervista a Vincenzo Consolo, in L'opera di Vincenzo Consolo. (Dissertazione inedita presentata presso la University of Alberta), 1992, pp. 35-49.

Geerts W., L'euforia a tavola. Su Vincenzo Consolo, in Soavi sapori della cultura italiana, Atti del XIII Congresso dell'A.I.P.I. Verona/Soave 2729 agosto 1998, B. Van den Bossche ed., Firenze, Cesati, 2000, pp. 307-316.

Nabokov V., Lolita, Novels 1955-1962, New York, The Library of America, 1996.

Scuderi A., Scrittura senza fine. Le metafore malinconiche di Vincenzo Consolo, Enna, Il Lunario, 1997.

Segre C./ Ossola C. ed., Antologia della poesia italiana. Duecento, Torino, Einaudi, 1997.

Traina G., Vincenzo Consolo, Fiesole (Firenze), Cadmo, 2001. 